\title{
ANÁLISE DA IMPLANTAÇÃO DA METODOLOGIA CERNE: ESTUDO DE CASO EM DUAS INCUBADORAS NUCLEADORAS DO PARANÁ
}

DOI: 1014211/regepe33007

\author{
Cristiane De Almeida - Universidade Federal do Paraná ${ }^{1}$ \\ Carmem Kistemacher Barche - Universidade Federal do Paraná2 \\ Andréa Paula Segatto - Universidade Federal do Paraná ${ }^{3}$
}

Resumo: Este artigo tem como objetivo analisar a implantação da Metodologia Centro de Referência para Apoio a Novos Empreendimentos (CERNE), criada pelo Serviço Brasileiro de Apoio às Micro e Pequenas Empresas (SEBRAE) em parceria com a Associação Nacional das Entidades Promotoras de Empreendimentos Inovadores (ANPROTEC), em duas Incubadoras do estado do Paraná, classificadas como Nucleadoras, ou seja, que já estão fortemente estabelecidas e possuem condições de fornecer apoio às demais incubadoras, objetivando o desenvolvimento coletivo. Além de uma pesquisa bibliográfica, foi realizado o levantamento de informações por meio de entrevistas com pessoas envolvidas no processo de implantação, contemplando questões relativas aos benefícios percebidos, as principais dificuldades encontradas no processo e, consequentemente, possibilidades e necessidades de estudos futuros sobre o tema. Os resultados levantados demonstram que as incubadoras identificam como vantagens, na implantação da metodologia CERNE, a visibilidade no mercado, melhoria na qualidade dos processos e padronização das atividades. Em relação às dificuldades, identificou-se que existem poucos profissionais especialistas e falta de indicadores padronizados. Este estudo refere-se ao levantamento da implantação da primeira etapa da metodologia - CERNE 1 - e pode ser base para estudos futuros com o intuito de identificar os impactos, bem como as melhorias necessárias.

Palavras-chave: Incubadora; CERNE; Processo de Incubação.

\section{ANALYSIS OF THE IMPLEMENTATION OF CERNE METHODOLOGY: CASE STUDY IN TWO NUCLEATINGS INCUBATORS FROM PARANÁ}

\begin{abstract}
This article aims to analyze the implementation of the Methodology Reference Center for Support to New Enterprises (CERNE), created by the Brazilian Service of Support for Micro and Small Enterprises (SEBRAE) in partnership with the National Association of the Promoter Organizations of Innovative Enterprises (ANPROTEC), in two incubators in the state of Paraná, classified as nucleation, that are already established and have strong position to provide support to other incubators, aiming the collective development. In addition to a literature search, was conducted a collection of information through interviews with

\footnotetext{
${ }^{1}$ E.mailcristianealm@gmail.com- Endereço: Av. Prof. Lothario Meissner, 632 2oandar - Jardim Botânico, Curitiba-PR. CEP: 80210-170.

2 E.mail: carmembarche@ufpr.br

${ }^{3}$ E.mail: aps@ufpr.br
}

ALMEIDA, C. de; BARCHE, C. K.; SEGATTO, A. P. Análise da implantação da metodologia Cerne - estudo de caso em duas incubadoras nucleadoras do Paraná. Revista de

Empreendedorismo e Gestão de Pequenas Empresas, v. 3, n. 3, p. 194-210, 2014. 
people involved in the deployment process, covering issues relating to perceived benefits, the main difficulties encountered in the process and, consequently, possibilities and needs for future studies on the theme. The results demonstrate that the incubators surveyed identify as advantages in the implementation of the methodology CERNE, visibility in the market, improving the quality of processes and standardization activities. In terms of the difficulties identified there are few specialists and lack of standardized indicators. This study refers to survey implementation of the first phase of the methodology - CERNE 1, and can be the basis for future studies in order to identify the impacts and improvements.

Keywords: Incubator; CERNE; Incubation Process.

\section{Introdução}

Um dos grandes desafios das empresas nascentes é criar estrutura e adquirir conhecimentos que lhes permitam crescer e permanecer no mercado cada vez mais competitivo. De acordo com informações do SEBRAE, no estudo Sobrevivência das Empresas no Brasil (2013), o índice de mortalidade das empresas nascidas em 2007 atingiu 24,4\%. Um percentual menor do que das empresas implantadas em 2005, porém ainda em um patamar elevado. Sobre as causas da mortalidade das empresas, o SEBRAE (2008) menciona não existir um motivo único, mas sim uma junção de fatores, como falta de comportamento empreendedor, ausência de planejamento, falhas no processo de gestão empresarial, insuficiência de políticas públicas de apoio, impactos ocorridos na economia como um todo, entre outros.

Diante desse contexto, os empreendedores, no início, podem buscar as incubadoras como forma de conseguir estruturar seu negócio. Porém, inúmeras vezes, erroneamente, alguns as visualizam apenas como uma estrutura física para desenvolver suas ideias.

De acordo com a ANPROTEC (2013), as incubadoras têm o objetivo de amparar o pequeno empreendedor fornecendo-Ihe, além da infraestrutura, o desenvolvimento da capacidade técnica, gerencial e administrativa. Assim, além de possuir competência para identificar negócios em potencial, a incubadora pode e deve oferecer estrutura física para o desenvolvimento de uma ideia ou projeto, mas também estruturação e preparação dos empreendedores para gerir seus negócios.

Corroborando a isso, Lalkaka (1996) ressaltou que incubadoras de empresas são caracterizadas por um ambiente de trabalho controlado, projetado 
para auxiliar no crescimento de novas empresas emergentes Sendo assim, tem-se a primazia de criar um clima cooperativo para o treinamento, suporte e desenvolvimento de empresas e empreendedores.

O Ministério da Ciência e Tecnologia - MCT (2001) ainda amplia a relevância da participação das incubadoras, afirmando que estas atuam como promotoras de desenvolvimento econômico e social, devendo atentar para as possibilidades de inovação tanto em produtos, quanto em processos e métodos. Para atender a esses objetivos, segundo a ANPROTEC (2013), a transformação de ideias em negócios de sucesso no processo de incubação ocorre na geração de informação e, acima de tudo, de conhecimento.

Mecena e Magacho (2007) relatam que uma das formas que o movimento de incubação assume é a de gerador de inovações a partir do desenvolvimento de empreendimentos, determinando assim, resultados econômicos e tecnológicos significativos no ambiente ou arranjo em que se insere.

Sobre a inovação, Tidd, Bessant e Pavitt (2008, p. 30) salientam: "sejam quais forem as condições tecnológicas, sociais ou mercadológicas envolvidas, a chave para se criar, e manter vantagem competitiva tende a pertencer àquelas organizações que inovam constantemente."

Ainda segundo os autores, a inovação é uma questão de conhecimento, que pode ser gerado mediante a capacidade de transformação das incertezas. E é na gestão das incertezas, ou seja, na preparação para enfrentá-las, que as incubadoras têm sua participação significativa. Ao entender os reflexos da atuação das incubadoras, fica claro que melhorias realizadas no processo de incubação influenciarão no sucesso dos novos negócios e, consequentemente, na economia de todo país.

A Metodologia CERNE surge, então, como uma base de referência que contribui nesse processo de melhoria, disponibilizando um escopo de execução de atividades para diminuir a variabilidade nas atividades, permitindo, além de crescimento quantitativo dos negócios, melhorias na qualidade.

ALMEIDA, C. de; BARCHE, C. K.; SEGATTO, A. P. Análise da implantação da metodologia Cerne - estudo de caso em duas incubadoras nucleadoras do Paraná. Revista de

Empreendedorismo e Gestão de Pequenas Empresas, v. 3, n. 3, p. 194-210, 2014.196 
As incubadoras de empresas

De acordo com dados da ANPROTEC (2013), tem-se uma elevação expressiva nos últimos dez anos no que concerne o movimento de incubadoras, alcançando em média um crescimento de $25 \%$, sendo que, as incubadoras brasileiras apoiam em torno de quatro mil e quinhentas empresas. Juntas faturam cerca de quatrocentos milhões de reais e geram aproximadamente vinte mil empregos diretos.

As incubadoras, de acordo com Medeiros (1998, p. 6-7), são consideradas arranjos interinstitucionais, contendo "instalações e infraestrutura apropriadas, estruturado para estimular e facilitar":

[...] A vinculação empresa-universidade (e outras instituições acadêmicas); o fortalecimento das empresas e o aumento de seu entrosamento; e o aumento da vinculação do setor produtivo com diversas instituições de apoio (além das instituições de pesquisa, prefeituras, agências de fomento e financiamento - governamentais e privadas - instituições de apoio às micro e pequenas empresas - como o Serviço Brasileiro de Apoio às Micro e Pequenas Empresas no Brasil - e outras (MEDEIROS, 1998, p. 6-7).

Neste sentido, Vedovello e Figueiredo (2005, p. 5), ressaltam que as incubadoras tem realizado um papel cada vez mais importante e sua estrutura possibilita "aglutinar políticas e atores sociais distintos e canalizar esforços e recursos com o objetivo de promover um ambiente econômico mais pró-ativo que favoreça o desenvolvimento socioeconômico mais sustentado e competitivo". Grimaldi e Grandi (2005) ainda complementam salientando que as incubadoras são meios eficazes de associar tecnologia, capital e know-how, alavancando a empresa e podendo acelerar seu desenvolvimento.

Apesar das incubadoras representarem contribuição amplamente significativa para a competitividade das empresas e desenvolvimento das regiões onde estão instaladas, conforme a ANPROTEC (2013), tem-se observado a necessidade das incubadoras sintonizarem suas estruturas e serviços às novas exigências da sociedade. Isto pela razão de que há mudanças nas formas e na natureza da competição, principalmente no sentido de que esta competição não é mais local, podendo ocorrer entre diferentes regiões.

ALMEIDA, C. de; BARCHE, C. K.; SEGATTO, A. P. Análise da implantação da metodologia Cerne - estudo de caso em duas incubadoras nucleadoras do Paraná. Revista de

Empreendedorismo e Gestão de Pequenas Empresas, v. 3, n. 3, p. 194-210, 2014. 
Sendo assim, a ANPROTEC salienta a importância de uma ampliação quantitativa e qualitativa dos resultados das incubadoras, alcançando um percentual mais expressivo da população. Mas para que isso ocorra, é essencial a implantação de um modelo de atuação que dê conta da complexidade desta nova realidade, reduzindo a variabilidade dos resultados alcançados. É neste novo contexto que se insere o modelo CERNE.

\section{Centro de Referência para Apoio a Novos Empreendimentos - CERNE}

O CERNE é uma metodologia desenvolvida em parceria pelo SEBRAE e ANPROTEC, com o objetivo de criar uma plataforma de soluções, de forma a ampliar a capacidade da incubadora em gerar empreendimentos inovadores bem sucedidos. Este modelo foi inspirado no modelo de apoio à micro e pequenas empresas existentes nos Estados Unidos, o Small Business Development Centers (SBDCs), que se refere a um programa de geração de oportunidades para pequenas empresas.

A ideia do CERNE é que incubadoras, de diferentes áreas e tamanhos, possam utilizar elementos básicos para garantir o sucesso das empresas apoiadas. Essa metodologia contempla três níveis de abordagem: a empresa, o processo de incubação e a incubadora. A metodologia está embasada em oito princípios, como pode ser observado na Figura 1. 


\section{A Revista da ANEGEPE

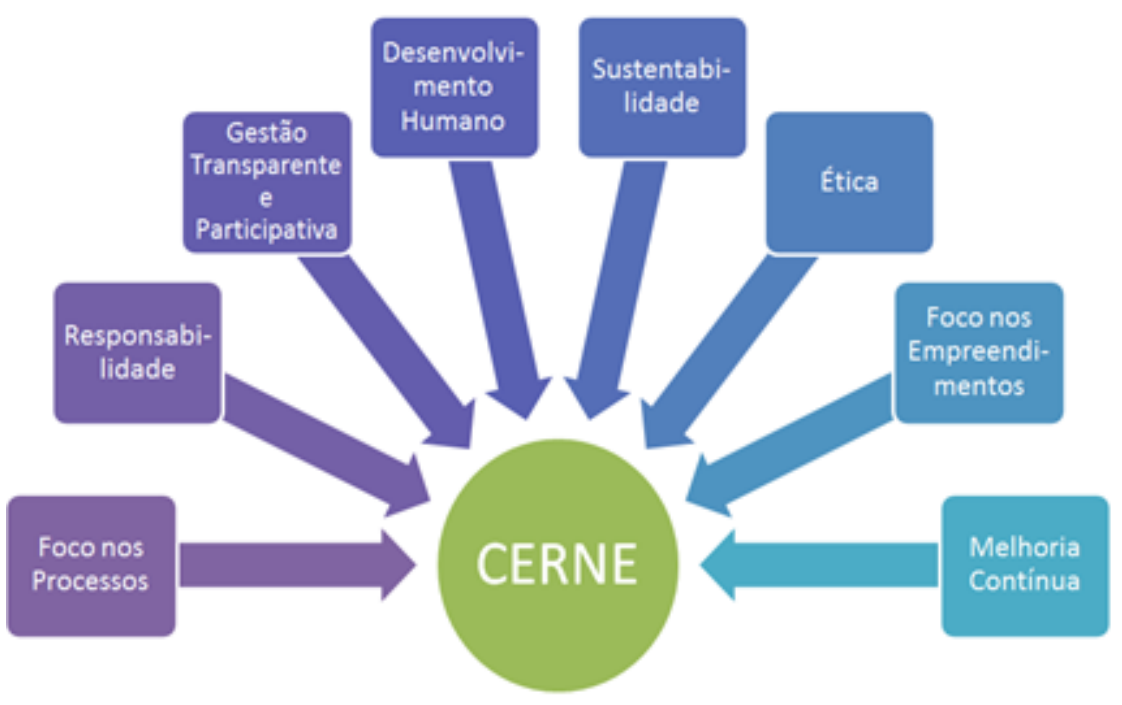

FIGURA 1 - Princípios Básicos do Modelo CERNE

Fonte: Centro de Referência para Apoio a Novos Empreendimentos - CERNE. (2013, p.6). Sumário Executivo. ANPROTEC, Brasília, DF, Brasil.

O CERNE busca proporcionar maturidade às incubadoras, sendo capazes de gerar sistematicamente empreendimentos de sucesso (ANPROTEC, 2013). Para isso, são trabalhados fatores como: aumento da transparência do processo de incubação, quantidade e qualidade de empreendimentos incubados, diminuição da variabilidade nos processos, entre outros.

A implantação da metodologia envolve um processo gradativo e contínuo que evolui à medida que as exigências são atendidas. Segundo a ANPROTEC, a estrutura de implantação está desenhada em 4 níveis, sendo:

- CERNE 1 - Empreendimento: Foco no processo de incubação e no desenvolvimento dos empreendimentos. Nesta etapa constam, por exemplo: sensibilização e prospeç̧ão, seleção, planejamento, qualificação, assessoria/consultoria, monitoramento, graduação e relacionamento com as graduadas, etc.

- CERNE 2 - Foco na incubadora como empreendimento: sistema de avaliação e certificação, sistema de geração de ideias, sistema de gestão estratégica, sistema de serviços e empreendimentos.

- CERNE 3 - Foco na consolidação e ampliação da rede de parceiros: sistema de apoio ampliado aos empreendimentos, sistema de monitoramento do desempenho da incubadora, sistema de participação no desenvolvimento regional sustentável. 
- CERNE 4 - Sistema de Melhoria Contínua.

O alcance dos CERNEs ocorre mediante a aplicação, comprovação e acompanhamento de processos e práticas-chave estabelecidos em cada etapa. Vale ressaltar que, além da evolução entre os CERNEs, existe uma evolução das fases de execução das atividades, que também ocorre de forma evolutiva. As fases nas quais se classificam as atividades são:

- Inicial: Quando é realizada e registrada;

- Definida: Quando é feita como parte de um planejamento;

- Estabelecida: Quando já ocorre o monitoramento, possui indicadores de medição;

- Sistematizada: Quando já foi realizada no mínimo uma reunião de análise crítica, verificação dos resultados e comparação dos indicadores.

A figura 2 apresenta o detalhamento das práticas-chave de cada CERNE, bem como as possibilidades de obtenção de cada um dos níveis.

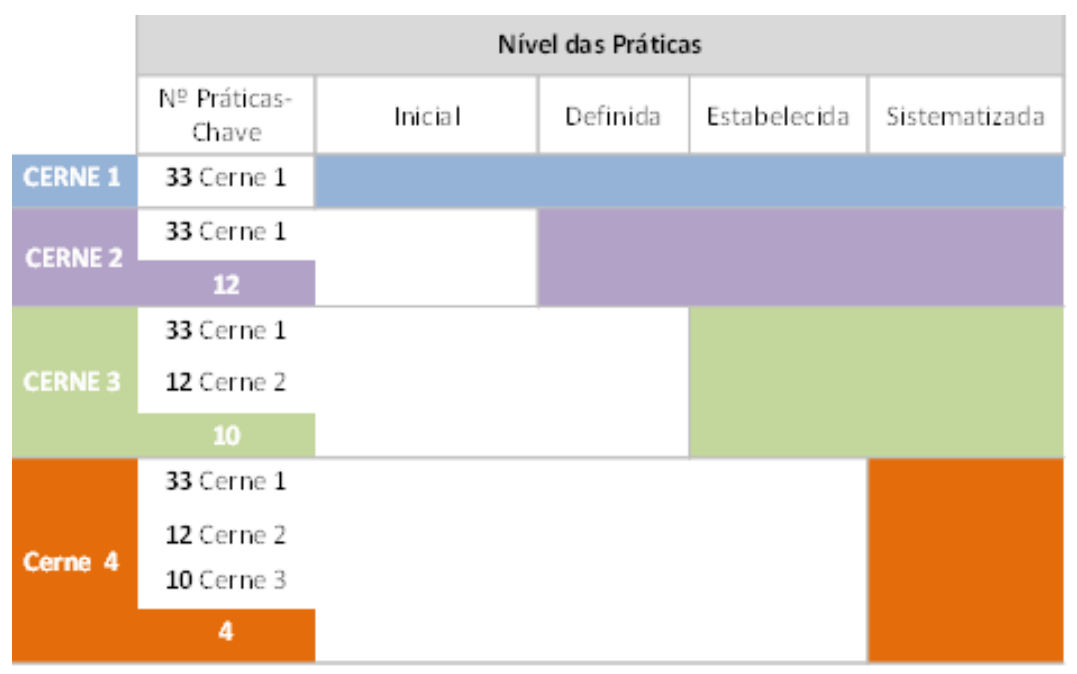

FIGURA 2 - Evolução da implantação CERNE

Fonte: Adaptado de Centro de Referência para Apoio a Novos Empreendimentos - CERNE. (2013). Sumário Executivo. ANPROTEC, Brasília, DF, Brasil.

O primeiro passo para a incubadora implantar a metodologia CERNE consiste na verificação e implantação das 33 práticas do CERNE 1. Vale ressaltar que a incubadora deve atender as práticas-chave do nível, para então ser considerada como detentora desse CERNE. Assim, se alguma prática-chave não é realizada, o CERNE não poderá ser obtido até que estejam realizadas as práticas correspondentes nos níveis exigidos. 
Para enquadramento no CERNE 1, por exemplo, a instituição deve apresentar diagnóstico, mencionando e comprovando realizar as 33 práticas-chave, podendo estas se encontrarem em qualquer um dos níveis, do inicial ao sistematizado.

Já para estar enquadrada no CERNE 2, a empresa necessariamente precisa atender as 33 práticas do CERNE 1, no mínimo de forma definida (com planejamento prévio) e incluir mais 12 práticas-chave do CERNE 2, e assim sucessivamente.

O CERNE 4 somente será alcançado quando as 59 práticas-chave constantes na metodologia forem atendidas de forma sistematizada. Nesse momento, acreditase que o processo de melhoria contínua estará instaurado em definitivo nas incubadoras e, consequentemente, nos negócios como um todo, pois as práticas estarão contemplando os negócios (incubados), a incubadora e a rede de parceiros. A melhoria contínua é fator primordial para o sucesso dos negócios e, nesse sentido, Blauth (2006, p.61) afirma que "um dos princípios da excelência é a avaliação e melhoria permanente dos processos".

O formato no qual foi estruturada a metodologia CERNE atende aos requisitos de melhoria contínua e por tratar-se de uma metodologia nova, existem algumas etapas em desenvolvimento e adaptação, estando o escopo geral definido. Porém as práticas-chave, finalizadas e divulgadas até o momento, referem-se apenas ao CERNE 1, e são apresentadas e detalhadas no Quadro 1. 


\begin{tabular}{|c|c|c|}
\hline \multicolumn{3}{|c|}{$\begin{array}{l}\text { REVISTA } \\
\text { Revista de Empreendedorismo } \\
\text { e Gestá de Pequenas Empresas }\end{array}$} \\
\hline $\begin{array}{c}\text { PROCESSO } \\
\text { CHAVE }\end{array}$ & PRÁTICA-CHAVE & OBJETIVO \\
\hline $\begin{array}{l}\text { Sensibilização e } \\
\text { Prospecção }\end{array}$ & $\begin{array}{l}\text { - Sensibilização; } \\
\text { - Prospecção; } \\
\text { - Qualificação de Potenciais } \\
\text { Empreendedores. }\end{array}$ & $\begin{array}{l}\text { Ampliar a quantidade, qualidade e } \\
\text { diversidade das propostas } \\
\text { apresentadas à incubadora. }\end{array}$ \\
\hline Seleção & $\begin{array}{l}\text { - Recepção de Propostas; } \\
\text { - Avaliação; } \\
\text { - Contratação. }\end{array}$ & $\begin{array}{l}\text { Garantir que sejam selecionados } \\
\text { empreendimentos inovadores, com } \\
\text { maior probabilidade de sucesso, e } \\
\text { garantir transparência no } \\
\text { relacionamento entre as partes. }\end{array}$ \\
\hline Planejamento & $\begin{array}{l}\text { - Plano de desenvolvimento do } \\
\text { Empreendedor; } \\
\text { - Plano Tecnológico; } \\
\text { - Plano de Capital; } \\
\text { - Plano de Mercado; } \\
\text { - Plano de Gestão. }\end{array}$ & $\begin{array}{l}\text { Garantir que os empreendedores } \\
\text { incluam os cinco eixos no } \\
\text { planejamento e desenvolvimento de } \\
\text { seu negócio. }\end{array}$ \\
\hline Qualificação & $\begin{array}{l}\text { - Qualificação nos Cinco Eixos } \\
\text { (Empreendedor, Tecnológico, } \\
\text { Financeiro, Mercado e Gestão). }\end{array}$ & $\begin{array}{l}\text { Promover a qualificação dos } \\
\text { empreendedores e colaboradores } \\
\text { nos cinco eixos norteadores do } \\
\text { negócio. }\end{array}$ \\
\hline $\begin{array}{l}\text { Assessoria } \\
\text { Consultoria }\end{array}$ & $\begin{array}{l}\text { - Assessoria e } \\
\text { Cinco Eonsultoria nos } \\
\text { Tecnológico, (Empreendedor, } \\
\text { Mercado e Gestão). }\end{array}$ & $\begin{array}{l}\text { Promover o desenvolvimento e } \\
\text { utilização de soluções que } \\
\text { contribuam para o crescimento dos } \\
\text { negócios. }\end{array}$ \\
\hline Monitoramento & $\begin{array}{l}\text { - Monitoramento dos Cinco Eixos } \\
\text { (Empreendedor, Tecnológico, } \\
\text { Financeiro, Mercado e Gestão). }\end{array}$ & $\begin{array}{l}\text { Avaliar e contribuir para o } \\
\text { desenvolvimento da maturidade nos } \\
\text { negócios. }\end{array}$ \\
\hline $\begin{array}{l}\text { Graduação e } \\
\text { Relacionamento } \\
\text { com Graduadas }\end{array}$ & $\begin{array}{l}\text { - Processo de Graduação; } \\
\text { - Relacionamento com } \\
\text { Graduadas. }\end{array}$ & $\begin{array}{l}\text { Garantir que o processo de saída da } \\
\text { empresa seja bem sucedido, } \\
\text { mantendo o vínculo e oferecendo } \\
\text { serviços agregados. }\end{array}$ \\
\hline $\begin{array}{l}\text { Sistema de } \\
\text { Gerenciamento } \\
\text { Básico }\end{array}$ & $\begin{array}{l}\text { - Modelo Institucional; } \\
\text { - Gestão Financeira } \\
\text { Sustentabilidade; } \\
\text { - Infraestrutura Física } \\
\text { Tecnológica; e } \\
\text { - Apoio à Gestão; } \\
\text { - Comunicação e Marketing. }\end{array}$ & $\begin{array}{l}\text { Garantir o funcionamento efetivo da } \\
\text { incubadora e a possibilidade de } \\
\text { realizar parcerias. }\end{array}$ \\
\hline
\end{tabular}

QUADRO 1 - Processos e Práticas-Chave CERNE 1

Fonte: Adaptado de Centro de Referência para Apoio a Novos Empreendimentos - CERNE. (2013). Sumário Executivo. ANPROTEC, Brasília, DF, Brasil.

A estrutura do CERNE 1 é composta por 8 processos, com suas práticaschave correspondentes e para alcançá-las, a incubadora deve estabelecer mecanismos de prospecção para aumentar o número de candidatos a serem incubados, definir critérios claros de seleção, verificar potencialidades e demais variáveis que possam influenciar no sucesso de um negócio. A incubadora deve, ainda, capacitar os empreendedores sobre como gerir o seu negócio de forma efetiva nos cinco eixos considerados primordiais (tecnológico, financeiro, mercado e

ALMEIDA, C. de; BARCHE, C. K.; SEGATTO, A. P. Análise da implantação da metodologia Cerne - estudo de caso em duas incubadoras nucleadoras do Paraná. Revista de

Empreendedorismo e Gestão de Pequenas Empresas, v. 3, n. 3, p. 194-210, 2014.202 
gestão), além de manter uma estrutura de gerenciamento básico da incubadora para garantir sua sustentabilidade.

$\mathrm{Na}$ implantação da metodologia CERNE, a incubadora passa a ensinar o empreendedor a como fazer a gestão do negócio, sem realizar, em momento algum, as atividades para ele. Assim, não se pensa no empreendedor somente no período que está incubando ou para estruturar seu negócio, mas principalmente na continuidade desse processo, depois de graduada.

Com o objetivo de incentivar e viabilizar as incubadoras, a implantação da metodologia CERNE, o SEBRAE e a ANPROTEC disponibilizaram recursos, por meio de um edital, para essa implantação.

\section{Edital SEBRAE/ANPROTEC}

O Edital no 01/2011 refere-se especificamente à implantação da metodologia CERNE, permitindo a participação de todas as incubadoras do país que poderiam concorrer a recursos financeiros para viabilizar o processo. Foram contempladas ao todo, 152 incubadoras, sendo 44 tipo 1 (Nucleadoras) e 108 tipo 2 (Nucleadas).

No que diz respeito ao apadrinhamento, o Edital SEBRAE/ANPROTEC no 01/2011, define:

\footnotetext{
Apadrinhar: Dar apoio às incubadoras de empresas que não apresentam, ainda, as condições necessárias para implementação autônoma do modelo CERNE.

Incubadora Nucleada (tipo 2): Incubadoras de empresas que serão apadrinhadas por incubadoras mais estruturadas na implementação do modelo CERNE.

Incubadora Nucleadora (tipo 1): Incubadoras de empresas que darão apoio às incubadoras que não apresentam, ainda, as condições necessárias para implementação autônoma do modelo CERNE.
}

Levando em consideração a estrutura da metodologia e o Edital específico lançado, este estudo baseia-se no processo de implantação realizado por duas incubadoras contempladas no edital, para verificação dos fatores relevantes. 
Metodologia

O presente trabalho é caracterizado como qualitativo e conforme menciona Creswell (2010), é uma pesquisa interpretativa, pois o pesquisador buscará envolver-se no processo e intensificar sua participação. Por tal constatação, Stake (2000) salienta que os pesquisadores qualitativos são hóspedes nos espaços privados do mundo, seus modos devem ser bons, seu código de ética rigoroso e, ainda que junto com um árduo trabalho, os pesquisadores qualitativos tem interesse intenso em pontos de vista pessoais e das circunstâncias.

Neste sentido, Vieira (2004, p. 15) corrobora ressaltando que a pesquisa qualitativa "atribui importância fundamental à descrição detalhada dos fenômenos e dos elementos que o envolvem, aos depoimentos dos atores sociais envolvidos, aos discursos, aos significados e aos contextos".

Trabalhou-se neste artigo, com o método de estudo de caso. Escolheu-se esta estratégia de estudo, pois, de acordo com Yin (2005), no estudo de caso, se faz uso de questões do tipo "como" e "por que", devido a estas serem mais explanatórias. É neste sentido de explanação e definição que este estudo contempla.

Para a coleta de dados do presente artigo, foram realizadas entrevistas direcionadas com duas incubadoras do estado do Paraná, contempladas pelo edital do SEBRAE/ANPROTEC como Nucleadoras e que estão em processo de implantação/certificação do CERNE 1. Utilizou-se também, a técnica de levantamento bibliográfico a respeito das incubadoras e da Metodologia CERNE.

O roteiro de execução das entrevistas continha informações sobre a estrutura da implantação: se foi criada equipe específica para este processo, quantas pessoas participam da atividade, se foram contratadas pessoas para auxiliar, também sobre as principais vantagens percebidas com a implantação da metodologia e, por fim, as dificuldades encontradas.

Cumprindo com o objetivo proposto por este trabalho, de avaliar a implantação da Metodologia CERNE (Centro de Referência para Apoio a Novos Empreendimentos), criada pelo Serviço Brasileiro de Apoio às Micro e Pequenas Empresas (SEBRAE) em parceria com a Associação Nacional das Entidades 
Promotoras de Empreendimentos Inovadores (ANPROTEC), tem-se, após a análise dos casos, algumas considerações e possibilidades de estudos e acompanhamentos futuros.

\section{Análise dos dados}

Ao iniciar a análise dos dados coletados nas entrevistas, percebe-se que as duas incubadoras entrevistadas são bem estruturadas, com tempo de atuação significativo. Ambas possuem o processo de incubação em seu escopo de atividades, empresas já graduadas e envolvimento no desenvolvimento de novos negócios.

A primeira incubadora analisada é a Incubadora Tecnológica de Curitiba INTEC, fundada em 4 de setembro de 1989, e liderada pelo Instituto de Tecnologia do Paraná (TECPAR). Situada em Curitiba/PR, a incubadora apoia o desenvolvimento de negócios de empresas de base tecnológica, trabalhando com duas modalidades de incubação: residentes (instaladas nas dependências da INTEC) e não residentes (não utilizam as dependências físicas da INTEC, usufruindo de apoios gerenciais e técnicos, e serviços de infraestrutura da incubadora).

A segunda incubadora analisada é o Parque Tecnológico Itaipu - PTI, criado em 2003 com o objetivo de realizar ações para estimular o desenvolvimento econômico por meio da criação e a consolidação de empresas baseadas em produtos, processos e serviços inovadores, impulsionando a geração de emprego e renda na região. Possui três etapas de desenvolvimento de negócios: préincubação, incubação empresarial e condomínio empresarial.

As duas incubadoras responderam as mesmas indagações, o que permitiu a triangulação e comparação das respostas, visando propiciar validade e confiabilidade à pesquisa, as questões foram transcritas e tabuladas em um quadro comparativo (Quadro 2), com o objetivo de identificar semelhanças e variações no processo de implantação da metodologia - CERNE 1. 
NUCLEADORAS

\begin{tabular}{|c|c|c|}
\hline \multirow[b]{2}{*}{ QUESTÃO } & \multicolumn{2}{|c|}{ NUCLEADORAS } \\
\hline & $\begin{array}{l}\text { PARQUE TECNOLÓGICO ITAIPU - } \\
\text { PTI }\end{array}$ & $\begin{array}{l}\text { INCUBADORA TECNOLÓGICA DE } \\
\text { CURITIBA - INTEC }\end{array}$ \\
\hline $\begin{array}{l}\text { Possui algum tipo } \\
\text { de Certificação de } \\
\text { qualidade? }\end{array}$ & Não. & Utiliza os critérios ISO 9000. \\
\hline $\begin{array}{l}\text { Estrutura para a } \\
\text { implantação da } \\
\text { metodologia }\end{array}$ & $\begin{array}{l}\text { - A equipe de implantação é a } \\
\text { mesma existente na incubadora. } \\
\text { - Essa equipe elaborou um novo } \\
\text { modelo de incubação e iniciou a } \\
\text { implantação. }\end{array}$ & $\begin{array}{l}\text { - A equipe de implantação é a mesma } \\
\text { existente na incubadora. } \\
\text { - Essa equipe elaborou um novo } \\
\text { modelo de incubação e iniciou a } \\
\text { implantação. }\end{array}$ \\
\hline $\begin{array}{l}\text { Etapas } \\
\text { cumpridas }\end{array}$ & $\begin{array}{l}\text { Foi elaborado o diagnóstico inicial } \\
\text { em julho de 2013, e constatou-se } \\
\text { que a incubadora atende aos } \\
\text { critérios CERNE 1, em vários } \\
\text { processos, porém, alguns de } \\
\text { maneira informal e não registrada } \\
\text { necessitando, dessa forma, que seja } \\
\text { feito o detalhamento/descrição, } \\
\text { controle e acompanhamento. }\end{array}$ & $\begin{array}{l}\text { Foi elaborado o diagnóstico inicial e } \\
\text { constatou-se que a incubadora atende } \\
\text { aos critérios CERNE em boa parte dos } \\
\text { processos-chave em nível 1, porém } \\
\text { alguns de maneira informal e não } \\
\text { registrada necessitando, dessa forma, } \\
\text { que seja feito } \\
\text { detalhamento/descrição, controle e } \\
\text { acompanhamento. }\end{array}$ \\
\hline $\begin{array}{l}\text { Justificativas para } \\
\text { implantação da } \\
\text { Metodologia } \\
\text { CERNE }\end{array}$ & 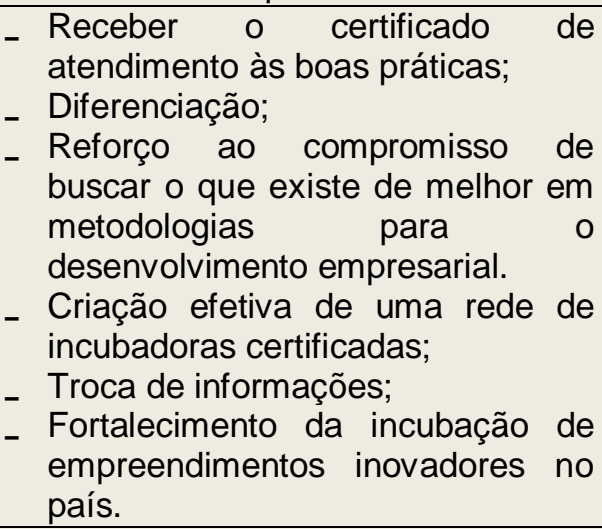 & $\begin{array}{l}\text { - } \text { Receber o certificado de } \\
\text { atendimento às boas práticas; } \\
\text { - Documentação e Padronização dos } \\
\text { processos realizados; } \\
\text { - Maior qualidade nos processos } \\
\text { realizados, com maior controle e } \\
\text { registro; } \\
\text { - Capacitação da equipe nos diversos } \\
\text { processos; } \\
\text { - Melhoria contínua nos processos. }\end{array}$ \\
\hline $\begin{array}{l}\text { Principais } \\
\text { Dificuldades } \\
\text { encontradas }\end{array}$ & $\begin{array}{l}\text { - Não possui uma metodologia } \\
\text { (indicadores) definida, ficando a } \\
\text { cargo de cada incubadora; } \\
\text { - Apesar de a metodologia propor } \\
\text { orientações quanto à melhoria dos } \\
\text { processos, ainda não atende ao } \\
\text { novo perfil das empresas mundiais, } \\
\text { especialmente às de tecnologia; } \\
\text { - Os consultores homologados não } \\
\text { possuem experiência adequada, } \\
\text { não entendendo em muitos casos } \\
\text { o funcionamento de uma } \\
\text { incubadora. }\end{array}$ & $\begin{array}{l}\text { - Mudança da gestão da incubadora } \\
\text { durante o processo de implantação; } \\
\text { - Por ser uma metodologia nova, } \\
\text { surgiram dúvidas quanto ao } \\
\text { entendimento das práticas; } \\
\text { - Equipe enxuta, necessitando } \\
\text { acumular atividades do dia a dia } \\
\text { com a implantação do CERNE; } \\
\text { - Falta de indicadores estabelecidos, } \\
\text { ficando a cargo da incubadora a } \\
\text { criação dos mesmos. }\end{array}$ \\
\hline Situação Atual & $\begin{array}{lcr}\begin{array}{l}\text { Atividades } \\
\text { aguardando } \\
\text { certificação. }\end{array} & \text { auditoria } & \text { andamento } \\
\text { para }\end{array}$ & $\begin{array}{l}\text { Atividades em andamento } \mathrm{e} \\
\text { aguardando auditoria para certificação. }\end{array}$ \\
\hline
\end{tabular}

QUADRO 2. Descrição das Entrevistas realizadas

Fonte: Elaborado pelas autoras (2013).

A análise do Quadro 2, demonstra que a INTEC utiliza critérios da ISO 9000, o que remete a um conhecimento prévio sobre manuais, procedimentos e fluxos. No

ALMEIDA, C. de; BARCHE, C. K.; SEGATTO, A. P. Análise da implantação da metodologia Cerne - estudo de caso em duas incubadoras nucleadoras do Paraná. Revista de

Empreendedorismo e Gestão de Pequenas Empresas, v. 3, n. 3, p. 194-210, 2014. 
item referente a etapas realizadas, ambas as incubadoras já realizaram diagnóstico inicial referente às práticas-chave do CERNE 1 , sinalizando que já realizam grande número de práticas, porém, em alguns casos, de maneira informal e não documentada. Nesta questão das práticas realizadas, as incubadoras se autodeclararam estarem em nível definido ou até mesmo sistematizado.

Quando perguntadas sobre os benefícios, as respostas das Nucleadoras foram semelhantes, mencionando considerarem relevante a implantação da metodologia para melhoria e padronização dos seus processos e obtenção de certificação de boas práticas.

A PTI sinaliza, ainda, como relevante, a criação de uma rede de incubadoras certificadas, o que proporcionaria ganhos em toda a cadeia e também permitiria uma maior diferenciação no mercado. Já a INTEC, adiciona a importância da capacitação da equipe nas práticas do CERNE 1, para nivelamento do conhecimento e elaboração de planos para desenvolvimento dos negócios nos cinco eixos empreendedor, tecnológico, capital, gestão e mercado.

Em se tratando das dificuldades, as respostas das duas incubadoras também são comuns, sendo fatores mencionados: falta de conhecimento sobre a metodologia tanto da incubadora como também de profissionais especialistas (consultores) e falta de indicadores padrões, sendo este requisito fundamental da metodologia quando menciona a obrigatoriedade de monitoramento.

Embora a ideia da metodologia seja dar liberdade para as empresas criarem seus próprios indicadores, não tendo um processo engessado, a falta de experiência transforma isso em uma dificuldade significativa para a implantação. Outro fator relevante das dificuldades encontradas, com o qual as incubadoras tiveram que se adaptar, certamente foi o referente à equipe.

Com a mesma estrutura existente na incubadora, a implantação da Metodologia CERNE surgiu como uma atividade extra, exigindo empenho dos participantes e adaptação das atividades diárias, para cumprimento das etapas necessárias. A INTEC ainda teve um agravante logo após a aprovação do edital, que foi a mudança na gestão.

As duas incubadoras estão em processo de implantação do CERNE 1 e realizaram além do diagnóstico inicial próprio, também o diagnóstico das Nucleadas, 
incubadoras menores que estão a elas alocadas e aguardam auditoria do SEBRAE/ANPROTEC para dar andamento à certificação.

\section{Considerações Finais}

O escopo da Metodologia CERNE desenvolvida pelo SEBRAE e ANPROTEC demonstra potencial de contribuir para melhorias nos processos de incubação. Conforme a leitura dos dados levantados nas duas incubadoras, existe interesse na implantação da metodologia, sendo observados benefícios a serem conquistados com essa ação.

Porém, por ser uma metodologia nova, observa-se que ainda são necessárias alterações e adequações no processo, principalmente no que tange a definição de equipes e apoio consultivo, como também à falta de dados históricos e maior conhecimento específico sobre dificuldades na implantação.

$\mathrm{O}$ fato de não possuir modelos definidos para monitoramento das práticas (indicadores padrões) configura uma dificuldade para as instituições, apontando a necessidade de uma estruturação básica para monitoramento e acompanhamento das atividades, o que viria a contribuir para a implantação da metodologia.

Embora a ANPROTEC tenha colocado à disposição treinamentos sobre a Metodologia CERNE, entende-se que ainda é necessário ampliar a quantidade de treinamentos realizados em busca de formar multiplicadores e especialistas para contribuir com a implantação e monitoramento do processo. Nesta questão, torna-se necessário que as pessoas que irão trabalhar com a implantação dessa metodologia, como consultores/profissionais desvinculados da incubadora, busquem absorver conhecimento específico da atividade de incubação.

Estudos futuros sobre essa necessidade e suas peculiaridades são uma das sugestões a serem feitas. Do mesmo modo, são necessários novos estudos sobre o tema para melhor aprofundar e solidificar o conhecimento acerca da metodologia, sua implantação e possíveis resultados.

Em se tratando das atividades programadas, além da avaliação para certificações do CERNE 1, torna-se necessário um monitoramento e 
acompanhamento dos resultados na implantação deste e dos demais CERNES, que possibilite identificar o desempenho e melhorias que se façam necessárias.

Por fim, salienta-se que um importante aspecto observado no estudo e que vem a colaborar para o sucesso da metodologia, é a importância das instituições que alcançarem a implantação e a certificação virem a contribuir com a implantação nas demais incubadoras, atuando como instituições de referência. Deste modo, a metodologia CERNE poderá desempenhar papel de relevância na elevação dos resultados positivos obtidos pelas incubadoras e na geração de novos negócios de sucesso.

\section{Referências:}

ANPROTEC. Sumário Executivo. Centro de referência para apoio a novos empreendimentos - CERNE, Brasília, DF, Brasil, 2013a.

ANPROTEC. Manual de Implantação do CERNE 1. Centro de referência para apoio a novos empreendimentos - CERNE, Brasília, DF, Brasil, 2013b.

ANPROTEC. Termo de Referência. Centro de referência para apoio a novos empreendimentos - CERNE, Brasília, DF, Brasil, 2013c.

BLAUTH, R. Gestão da Qualidade. Curitiba: IESDE Brasil, 2006. 544 p.

CRESWELL, J. W. Projeto de pesquisa: métodos qualitativo, quantitativo e misto. 3 ed. Porto Alegre:Artmed, 2010. 296 p.

GRIMALDI, R.; GRANDI, A. Business incubators and new venture creation: an assessment of incubating models. Technovation, v. 25, n. 2, p. 111-121, fev. 2005.

INCUBADORA TECNOLÓGICA DE CURITIBA - INTEC. Desafios do empreendedorismo tecnológico inovador. INTEC $20+20$, Curitiba, 2009.

LALKAKA, R. Technology business incubators: Critical determinants of success. Annals of The New York Academy of Sciences, Nova York, v. 798, p. 270-290, dez. 1996.

MECENA, S.; MAGACHO, L. Gestão de Competências em Redes de Organizações e Governança de Redes e de Arranjos: o que se discute na Academia e como usar na prática. In: Anais do encontro ReINC, 10, 2007, Rio de Janeiro. Anais... Rio de Janeiro, 2007. MEDEIROS, J. A. Incubadoras de empresas: Lição da experiência internacional. Revista de Administração, São Paulo, v. 33, n. 2, p. 5-20, abr./jun. 1998.

ALMEIDA, C. de; BARCHE, C. K.; SEGATTO, A. P. Análise da implantação da metodologia Cerne - estudo de caso em duas incubadoras nucleadoras do Paraná. Revista de

Empreendedorismo e Gestão de Pequenas Empresas, v. 3, n. 3, p. 194-210, 2014. 
MINISTÉRIO DA CIÊNCIA E TECNOLOGIA - MCT. Empresas graduadas nas Incubadoras Brasileiras em 2001. MCT, Distrito Federal, DF, Brasil, 2001.

PARQUE TECNOLÓGICO ITAIPU. Relatório Anual de atividades. Disponível em: <www.pti.org.br>. Acesso em: 20 ago. 2013.

SEBRAE. Inovação e Sustentabilidade: Bases para o Futuro dos Pequenos Negócios. Seminário Internacional sobre Pequenos Negócios, São Paulo, SP, Brasil, 2012.

SEBRAE. 10 Anos de Monitoramento da Sobrevivência e Mortalidade de Empresas. Sebrae-SP, São Paulo, 120 p., 2008.

SEBRAE. Sobrevivência das Empresas no Brasil. UGE-Sebrae, Brasília, DF, Brasil, 2013.

STAKE, R. Case studies. In: Cenzin, N. K.; Lincoln, Y.S. (eds.) Handbook of qualitative research. London: Sage, 2000.

TIDD,J.; BESSANT, J.; PAVITT, K. Gestão da Inovação. 3 ed. Porto Alegre, Bookman, 2008. 593 p.

VEDOVELLO, C.; FIGUEIREDO, P. N. Incubadora de Inovação: Que nova espécie é essa? RAE-eletrônica, v. 4, n. 1, art. 10, jan./jul. 2005.

VIEIRA, M. M. F. Por uma boa pesquisa (qualitativa) em Administração. In: VIEIRA, M.M.F.; ZOUAIN, D. M. (Org.). Pesquisa Qualitativa em Administração. Rio de Janeiro: Editora FGV, 2004.

YIN, R. K. Estudo de caso: planejamento e métodos. 3. ed. Porto Alegre: Bookman, 2005. 205 p.

Artigo recebido em: 26/11/2013. Artigo aprovado em: 16/10/2014

ALMEIDA, C. de; BARCHE, C. K.; SEGATTO, A. P. Análise da implantação da metodologia Cerne - estudo de caso em duas incubadoras nucleadoras do Paraná. Revista de 